BULL. AUSTRAL. MATH. SOC.

VOL. 36 (1987) 333-335
10F05, 10F40, 10K05, $10199,92 \mathrm{AO} 5$

\title{
Number sequences and phyllotaxis
}

\author{
TONy van RAVEnStein
}

This thesis is largely concerned with the study of phyllotaxis (leaf arrangement) of plants. Most higher order plants exhibit a remarkable degree of regularity in the positioning of their leaves. In a sunflower, for instance, one can perceive two sets of opposed spirals which each partition the set of florets. Intriguingly, the numbers of spirals are almost certainly consecutive members of the Fibonacci sequence $\left(F_{n}=F_{n-1}+F_{n-2}, n \geq 2, F_{0}=0, F_{1}=1\right)$. This pattern manifests itself in $95 \%$ of those plants which produce their leaves sequentially.

We begin our study by considering the distribution of $N$ points placed around the circle by a constant angle $a$. We might view this as a simplistic model of plant growth with each point representing a leaf appearing on a meristematic ring. The theory of continued fractions is used in offering a new proof of the steinhaus conjecture, a result which states that for any $\alpha$ and any $N$, the circle is partitioned into arcs or gaps of at most three, and at least two, different lengths.

Various means of measuring the degree of uniformity of this sequence of points are examined, providing evidence of the supremacy of the golden number, $\tau=(\sqrt{5}-1) / 2$. One such measure which we analyse is the

Received 24 October 1986. Thesis submitted to University of Wollongong, May 1986. Degree approved September 1986. Supervisor: Dr. Keith Tognetti.

Copyright Clearance Centre, Inc. Serial-fee code: 0004-9727/87 $\$ A 2.00+0.00$. 


\begin{abstract}
"discrepancy" of sequences, a quantity studied in the theory of uniform distribution of sequences modulo one (see Kuipers and Neiderreiter [4]). Two forms of this measure are actually considered, a relationship discovered between them, and some values of the two discrepancies are obtained using the theory of continued fractions (and the steinhaus conjecture).
\end{abstract}

We investigate further the manner in which the circle is divided by examining the transition from a sequence of gaps of two different lengths to another two-gap sequence. The problem is approached by labelling a gap according to its length (either large or small) - the sequence of strings thus obtained is analysed and, for the particular case where $a$ has identical terms in $i$ ts continued fraction expansion, a curious relationship is found between this sequence and the Bernoulli sequence. This latter sequence is $d_{1}, d_{2}, d_{3}, \ldots$ where $d_{i}=[(i+1) a]-[i a]$, first studied by Johann Bernoulli [3]. Particular attention is focused on the strings produced by $a=\tau$.

Higher dimensional models of phyllotaxis are considered. In particular, we study a phyllotaxis system arranged on the surface of a cylinder, with points lying at equal angular intervals on a regular helix. We determine the phyllotaxis system which ensures that the distance between neighbouring points is maximal, while the pitch of the spiral decreases during the process of growth. This is the contact pressure model considered by Adler [1], [2], a quantitative formulation of an hypothesis proposed by Schwenender [5] in 1878 to be the mechanism of Fibonacci phyllotaxis. We verify the consequences of his model by considering the analogous situation of contact pressure on the circle and extending the analysis to the cylinder.

In closing, we offer a model of phyllotaxis applicable to a general class of surfaces. We replace the condition that successive divergence angles be constant by the less restrictive assumption that the phyllotaxis system be "regular". The model simulates the emergence of Fibonacci phyllotaxis and has the advantage of being simple as well as biologically plausible. 


\section{References}

[1] I Adler, "A model of contact pressure in phyllotaxis", J. Theoret. Biol. 45 (1974), 1-79.

[2] I. Adler, "The consequences of contact pressure in phyllotaxis" J. Theoret. Biol. 65 (1977), 29-77.

[3] J. Bernoulli III, Sur une nouvelle espece de calcul. Recueil pour les Astronomes. T.I. (Vols. 1, 2. Berlin, 1772).

[4] I. Kuipers and H. Niederreiter, Uniform distribution of sequences (John Wiley and Sons, New York, 1974).

[5] S. Schwenender, Mechanische Theorie der Blattstellungslehre (Wilhelm Engelmann, Leipzig, 1878).

Department of Mathematics, University of Wollongong, Wollongong,

New South Wales, 2500

Australia. 